\title{
The impact of head repositioning accuracy and proprioception on cervical stabilization exercise in healthy adults
}

\author{
Kyung Wook Kang, Dae Won Kang, Gu Ye Kwon, Han Byul Kim, Kyoung Min Noh, Gi Hyun Baek, Jin Kwan Cha, \\ Hyun Hee Kim
}

Department of Physical Therapy, Shinsung University, Dangjin, Republic of Korea

\begin{abstract}
Objective: Cervical stabilization exercises are frequently to improve strength and endurance of cervical muscles. The purpose of this study was to identify changes in head repositioning accuracy (HRA) and neck proprioception through cervical stabilization exercises in healthy adults.

Design: One group pretest-posttest design.

Methods: Thirteen participants with no previous history of neck pain or injury to the cervical spine were recruited. HRA was measured by equipment including laser pointer, helmet, eye patch and marking pens. The distance between the spot where the beam had stopped and the center of the graph paper was measured three times with the averaged value used as the head repositioning accuracy. Neck proprioception was measured by a cervical range of motion device (CROM). Subjects wore the CROM tester and were to look straight ahead while bending his/her neck. Subjects were instructed to perform extension, lateral flexion and rotation, and the values were then measured and recorded. The measurements were performed pre-intervention, and after cervical stabilization exercise.
\end{abstract}

Results: There was no significant difference on HRA after intervention. In addition, there was no significant difference on neck proprioception compared with pre-intervention.

Conclusions: The present study did not identify any effect on HRA and neck proprioception of cervical stabilization exercise. Further investigations are required to elucidate this in old aged participants and patients with neck pain.

Key Words: Exercise, Neck, Proprioception, Proprioception

\section{Introduction}

A lot of people in this modern society have a musculoskeletal disorder in their neck and shoulder due to daily use of computer, including frequent use of mobile phone. Especially, postural stability is an essential factor in physical therapy as the changes in posture lead to the change in muscle activities or load on each joint according to the positions of joints related to alignment of human body, and the changes also have impacts on musculoskeletal system [1].

When a man is standing or sitting with his upper part of back hunched up, the position of his neck and head changes as a compensatory action [2]. The change in position of head may cause unbalance in the arrangement of musculoskeletal system, tension in neck-extensor muscles and weakness of neck-flexor muscles [3]. If this abnormal motor control continues for a long time, the joints or skeletons will not be able to make a normal movement with dynamics and physiological control [4].

The terminology 'proprioception' was used for the first time by Sherrington [5], who adopted the word to explain the sense of position and motion that were delivered by receptors located deep in muscles, tendons and joints. In the early days, the word was used almost in the same way as

Received: 8 May, 2015 Revised: 11 June, 2015 Accepted: 11 June, 2015

Corresponding author: Hyun Hee Kim

Department of Physical Therapy, Shinsung University, 1 Daehak-ro, Jeongmi-myeon, Dangjin 343-861, Republic of Korea

Tel: 82-41-350-1351 Fax: 82-41-350-1355 E-mail: ksuccess71@shinsung.ac.kr

(c) This is an Open-Access article distributed under the terms of the Creative Commons Attribution Non-Commercial License (http://creativecommons.org/licens es/by-nc/4.0) which permits unrestricted non-commercial use, distribution, and reproduction in any medium, provided the original work is properly cited.

Copyright $@ 2015$ Korean Academy of Physical Therapy Rehabilitation Science 
myesthesia, however, these days, it is used to express something complicated, including various types of senses, such as the sense of position, acceleration, perception of mobility and force [6].

Lederman et al. [7] reported that damages on muscles or joints may bring about a dysfunction on muscle spindle, and the proprioception could decrease according to a structural change caused a chemical change or damage around the muscle spindle. If there is damage to the proprioception, it will be difficult to make a proper response based on the collaboration with related muscles [8]. The proprioception may be divided into muscle, skin and joint proprioception, and it is the information obtained from joints, tendons, ligaments, muscles and skin. The muscle spindle and Golgi tendon organ are the dynamic receptor of muscles and tendons, and the main function of muscle spindle is to accept the changes in length of muscle and the speed of change, which are delivered to the nervous system. The main function of Golgi tendon organ is to detect the changes in tension between muscle-tendon units $[9,10]$. It is well known that most of proprioceptive receptors are located in tendons and articular capsules, and they are activated a lot at the anatomical end range [6]. Cuomo et al. [11] reported that the reduction of proprioceptive information led to the reduction of activation level of the surrounding muscles than controlled the joints.

Spinal stabilization is related to a proper response to the information regarding spine protection and stabilization, and the information is obtained from all sensory organs, such as the passive subsystems, active subsystems, and the control subsystem [12]. The range where resistance of joint is minimized or removed during a passive movement is defined as a neutral zone, and reduction of minute injury is very important for clinical stability of spine, so the shrinkage of this zone, which is caused by various damages or changes, may lead to a restriction on physiological movements [13]. The basic theory of stability exercises is related to maintaining neutral position to avoid the process to become disabled, which starts with minute injuries and degeneration of structures, as the various structures forming spine are worn out in routine activities, so it may be avoided by the exercise that helps stabilize spine through increasing flexibility, collaboration, endurance and muscle strength [14].

The cervical stabilization exercise can be used as a treatment that promotes body balance and function through control over neck joint movements for the patients with neck movement disorder, so the roles of muscle is emphasized in stability of cervical vertebra [15]. And recently, the cervical stabilization exercise is frequently used as an exercise that improves the muscle strength and endurance of neck muscles attached to the cervical vertebra [16]. The cervical vertebra is surrounded by the muscle that facilitates the head and neck movement, and the muscles in-depth, which are attached to each segment are working at all times regardless of the direction of movement. The longus capitis and longus colli play an important role in controlling and maintaining the neck movement [17], and unlike the sternocleidomastoid muscle and middle scalenus muscle, they are directly attached to the cervical vertebra as they are located in-depth [18]. Many neck muscles have certain number of muscle spindles per unit, so it can be assumed that the muscles are requested to have high-level function of proprioception [19]. Boyd-Clark et al. [18] reported that the longus colli has the higher distribution rate of muscle spindle than the multifidus muscle, so the roles of longus colli in postural stability has been more emphasized recently. And it has been argued that the static muscular endurance with low-level strength will play a more effective role in maintaining the cervical stabilization than the muscular contractile force of the neck flexor muscle in-depth [20]. Though we cannot measure the function of in-depth neck flexor muscle by carrying out a head-neck bending test, we may help keep the neck flat gradually by reducing the forward bending with contraction of longus colli after placing a pressurizer filed with air behind the neck. This is not designed to bend the head, but to bend the neck, so it is suitable to evaluate the anatomical activities of deep muscle rather than the activities of surface muscles $[17,21]$.

Therefore, this study aims to identify the influence of cervical stabilization exercise, in which the pressure biofeedback unit is used, on the head repositioning accuracy and proprioception in the students who have to sit on a chair to study or use a computer for a long time.

\section{Methods}

\section{Subjects}

The participants in this study consist of 13 college students participating in this program voluntarily. The candidates who had an external injury or neurologic damage around neck were excluded, because such injuries could have an impact on the study results. The average age of participants was 24.31 years, and their average height and weight were $170.61 \mathrm{~cm}$ and $62.69 \mathrm{~kg}$, respectively. 


\section{Outcome measure}

The head repositioning accuracy and neck proprioception was measure by using a cervical range of motion (CROM) tester (Sammons Preston Inc., Bolingbrook, IL, USA). The CROM tester has been devised to measure the CROM, and it helps carry out an objective measurement of neck flexion, extension, lateral flexion, and rotation.

\section{Procedures}

In order to measure the head repositioning accuracy, a 1 $\mathrm{m} \times 1 \mathrm{~m}$ graph paper was attached on the wall that was $2 \mathrm{~m}$ away from the subject. The subject was asked to sit upright on a chair put in accordance with the central line of on a graduated ruler. After wearing a helmet with a laser pointer attached on it, the subject was asked to move his/her head in order to make the beam be aimed at the center of the graph paper. And then the subject was asked to carry out a preliminary training five times in total. After completing the preliminary training, the experimenter asked the subject to aim the beam at the center of graph paper after turning the subject's head to the right. The experimenter measured the distance between the spot where the beam had stopped and the center of the graph paper, which was carried out three times, and then the average value was used as the head repositioning accuracy [22]. The less deviation implied that the head repositioning accuracy had got better. The experimenter asked the subject to stare forward naturally, and marked the point on the graph paper, which was in accordance with the laser beam. And the subject was instructed to stare forward naturally again after carrying out flexion, extension, lateral flexion and left-right rotation of head. The experimenter marked the point where the beam was shot, and the distance between the point marked in the first attempt and the other

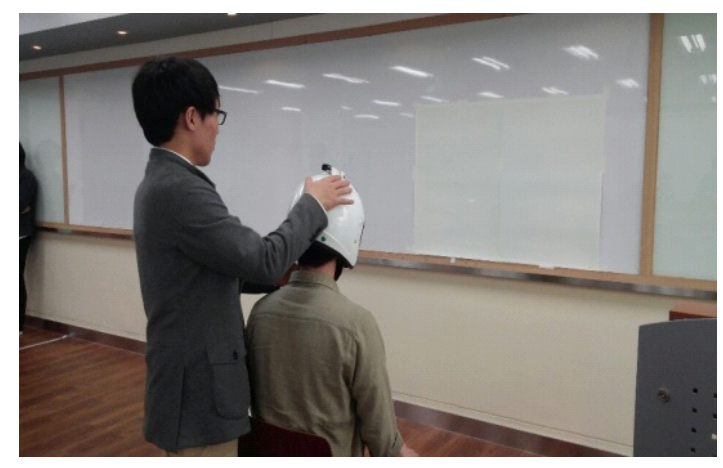

Figure 1. Evaluation of head repositioning accuracy. point marked in the second attempt by using the $\mathrm{x}$-axis and $\mathrm{y}$-axis (Figure 1).

In order to measure the proprioception of neck, the subject was instructed to stand with his/her feet shoulder length apart, and sit upright on the chair without leaning over the chair. The subject was asked to wear the CROM tester, looking straight ahead. The angle meters attached at the front, side and top of CROM tester were set at zero. And the subject was asked to bend his/her neck at an angle of 30 degrees to hold the pose for 3 seconds. And then the subject asked to remember the point of flexion. And after coming back to the neutral position, the subject was asked to go back to the previous position to keep the position for 3 seconds. The experimenter measured and recorded the flexion angle. In the method mentioned above, the subject was instructed to carry out the movements such as extension, left and right lateral flexion, and left and right rotation, and the measured values were recorded (Figure 2).

The cervical stabilization exercise was carried out by using the Stabilizer Pressure Biofeedback unit (Chattanooga

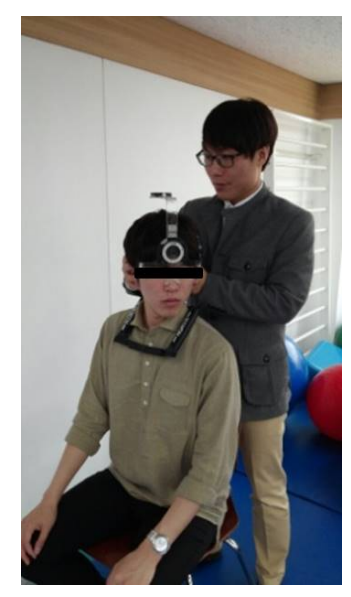

Figure 2. Evaluation of proprioception on the neck.

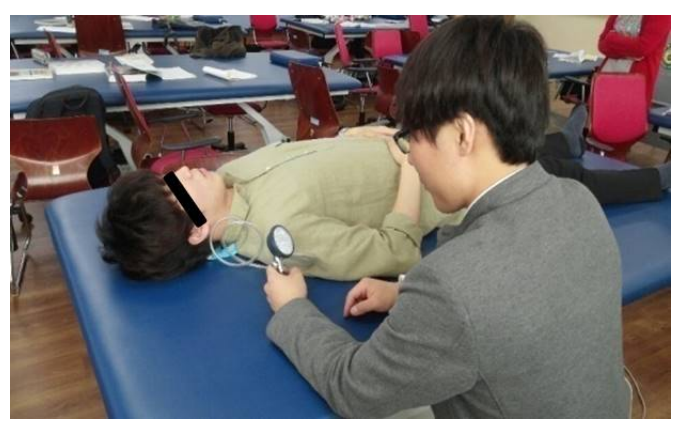

Figure 3. Cervical stabilization exercise. 
Table 1. Comparison of head repositioning accuracy (HRA) and proprioception at pre and post intervention $(\mathrm{N}=13)$

\begin{tabular}{|c|c|c|c|c|}
\hline Variable & Pre-intervention & Post-intervention & $t$ & $p$ \\
\hline \multicolumn{5}{|l|}{ HRA } \\
\hline $\mathrm{X}$-axis difference $(\mathrm{cm})$ & $8.15(6.19)$ & $5.23(3.79)$ & 1.502 & 0.159 \\
\hline Y-axis difference $(\mathrm{cm})$ & $15.62(13.84)$ & $7.69(6.17)$ & 1.821 & 0.094 \\
\hline \multicolumn{5}{|l|}{ Neck proprioception } \\
\hline Flexion $\left(^{\circ}\right)$ & $29.92(3.04)$ & $29.77(0.51)$ & 0.163 & 0.874 \\
\hline Extension $\left(^{\circ}\right)$ & $30.85(1.41)$ & $30.00(1.41)$ & 1.677 & 0.119 \\
\hline Right side-flexion $\left({ }^{\circ}\right)$ & $29.77(1.01)$ & $30.00(0.82)$ & -0.898 & 0.387 \\
\hline Left side-flexion $\left({ }^{\circ}\right)$ & $29.46(1.61)$ & $30.08(1.04)$ & -1.336 & 0.206 \\
\hline Right rotation $\left({ }^{\circ}\right)$ & $32.08(2.90)$ & $30.31(1.38)$ & 1.714 & 0.112 \\
\hline Left rotation $\left({ }^{\circ}\right)$ & $30.92(1.85)$ & $30.23(1.09)$ & 1.389 & 0.190 \\
\hline
\end{tabular}

Values are presented as mean (SD).

Medical Supply Inc., Hixson, TN, USA) (Figure 3). In the cervical stabilization exercise, the subject was lying on the table on his/her back, and the stabilizer was placed behind the neck, and then the stabilizer was swollen up to $20 \mathrm{mmHg}$ by inserting air. The subject was asked to tuck his/her chin and press the stabilizer placed behind the neck, so that the pressure could be kept at $30 \mathrm{mmHg}$ [10]. The subject was asked to keep the pose for 10 seconds at a time. And 3 sets of tests were carried, and each set was composed of 10 tests. Between each test the subject took 10-second break, and between each set the subject took 1-minute break.

\section{Data analysis}

In order to identify the head repositioning accuracy and proprioception of neck before and after the cervical stabilization exercise, the paired t test was carried out. And in order to verify the statistical significance, the level of significance was set at 0.05 , and the collected data was analyzed by using the SPSS ver. 12.0 for Windows (SPSS Inc., Chicago, IL, USA), a commercial statistical program.

\section{Results}

The head repositioning accuracy appeared to be reduced as the difference in $\mathrm{x}$-axis that had been $8.15 \pm 6.19 \mathrm{~cm}$ before the cervical stabilization exercise changed into $5.23 \pm 3.79$ $\mathrm{cm}$ after the exercise, and the difference in $y$-axis hat had been $15.62 \pm 13.84 \mathrm{~cm}$ changed into $7.69 \pm 6.17 \mathrm{~cm}$ after the exercise, however, there was no statistical significance. And the proprioception of neck appeared not to have a significant difference before and after the cervical stabilization exercise (Table 1).

\section{Discussion}

Neck pain, along with low back pain, is regarded as one of the most general factors causing dysfunction in everyday life, and the people in this modern society using a computer or mobile phone frequently cannot avoid the problem. As the neck shall have both the stability to support the head and dynamic characteristics related to the motility that helps the connectivity between head and body, so the habitual bad posture and repeated minute injuries may result in problems. When keeping the postural stability of neck, the roles of deep flexion muscle, longus colli and longus capitis are emphasized most frequently [18].

Chronic neck pain patients frequently face formation of straightened neck of backward flexion due to the structural deformation of cervical vertebra, and the deformation may result in neck pain. Taimela et al. [23] reported that the head repositioning accuracy of chronic neck pain patients was lower than that of normal person, and they argued that the hypoesthesia might have a negative effect on proper neck arrangement.

In this study, it was found out that a certain amount of intervention helped reduce the problem related to head repositioning accuracy, but the statistical significance has not been identified. Kim et al. [24] identified the significant difference in the ability to relocate head by applying a cervical stabilization exercise, where a sling was used. The exercise was carried out 24 times in total and the 50-minute exercise was carried out three times a week for 8 weeks. Kim et al. [25] also identified a significant result related to the sense of head relocation by carrying out the 30 -minute cervical stabilization exercise three times a week, and the exercise was carried out for 8 weeks in total. However, in this study, the 
head repositioning accuracy appeared to be reduced as the difference in $\mathrm{x}$-axis that had been $8.15 \pm 6.19 \mathrm{~cm}$ before the cervical stabilization exercise changed into $5.23 \pm 3.79 \mathrm{~cm}$ after the exercise, and the difference in $y$-axis hat had been $15.62 \pm 13.84 \mathrm{~cm}$ changed into $7.69 \pm 6.17 \mathrm{~cm}$ after the exercise, however, there was a difference from the precedent studies as no statistical significance could be identified. It is assumed that the difference from the precedent studies might have occurred because this study was aimed at bringing about a result from one cross-sectional study, while the precedent studies had been carried out by applying 8-week intervention.

The previous study results were based on the cervical stabilization exercise that had been carried 3 sets a day, 3 days a week for 5 weeks in total, identified that the sense of joint position among the kinds of proprioception increased significantly when coming back to the neutral position after the subjects from experimental group moved their neck to the left, right and upward as much as possible [26]. However, Lee [26] could not identify any significant difference in the control group who had taken a neck posture exercise and the Mckenzie exercise for an hour. This study has not identified any significance in the flexion, extension, lateral flexion to the right and left in comparison with the state before intervention. Jung et al. [27] confirmed that the sense of joint position, when bending and extending the neck at a sitting posture, had significantly less deviation in the upright posture than the bending posture. Though the present study was aimed at promoting the proprioception by recovering the normal posture of neck joint through the cervical stabilization exercise, it could not identify the change in promotion due to the too short term of exercise.

This study could not identify the effects of short-term cervical stabilization exercise on the changes in head repositioning accuracy and proprioception. However, if it is allowed the subjects to have enough time to do the cervical stabilization exercise like the precedent studies, we may assume that the neck will respond to the location of head through improvement of proprioceptive sensation as the information helping keep a correct posture is provided continuously, which may result in good effects on posture correction for body and neck.

The limitation of this study is that the term of cervical stabilization exercise was too short to cause a change through the exercise, and the participants were so healthy and young that it was difficult for them to feel the problems related to head repositioning accuracy and proprioception, which led to the difficulty in identifying the effects of cervical stabilization exercise.

This study aims to identify the impacts of cervical stabilization exercise of healthy adults on the head repositioning accuracy and proprioception. This study could not identify a significant change in the head repositioning accuracy and proprioception. And it could not identify the effects of cervical stabilization exercise because this study was aimed at identifying the change through a short-term exercise program based on a cross-sectional study. Therefore, it is expected that the effects of cervical stabilization exercise will be carried out for a longer-term exercise period, applying the procedure to various subjects with neck joint dysfunction.

\section{Conflict of Interest}

The authors declared no potential conflicts of interest with respect to the authorship and/or publication of this article.

\section{References}

1. Moon SE. General coordinative manipulation. 1st ed. Seoul: Jungdam Media; 2004.

2. Kendall FP, McCreary EK, Provance PG, Rodgers MM, Romani WA. Muscles: testing and function with posture and pain. 5 th ed. Baltimore: LWW; 2005.

3. Burgess-Limerick R, Plooy A, Ankrum DR. The effect of imposed and self-selected computer monitor height on posture and gaze angle. Clin Biomech (Bristol, Avon) 1998;13:584-92.

4. Kim SY, Lee HJ. Literature review on the association between a cervical dysfunction and the change of neuromuscular control activity. J Korean Acad Orthoped Man Ther 2006;12:57-67.

5. Sherrington CS. On the proprioceptive system, especially in its reflex aspect. Brain 1907;29:467-82.

6. Gandevia SC, Burke D. Does the nervous system depend on kinesthetic information to control natural limb movements? Behavioral and Brain Sciences 1992:15:614-32.

7. Lederman E, Breen AC, Hartman LS, Newham DJ. Fundamentals of manual therapy: physiology, neurology, and psychology. 1st ed. New York: Churchill Livingstone; 1997.

8. Shankar K. Exercise prescription. 1st ed. Philadelphia: Hanley \& Belfus; 1999.

9. Edin B. Cutaneous afferents provide information about knee joint movements in humans. J Physiol 2001;531:289-97.

10. Kisner C, Colby LA. Therapeutic exercise: foundations and techniques. 4th ed. Philadelphia: FA Davis; 2002.

11. Cuomo F, Birdzell MG, Zuckerman JD. The effect of degenerative arthritis and prosthetic arthroplasty on shoulder proprioception. J Shoulder Elbow Surg 2005;14:345-8.

12. Panjabi M, Abumi K, Duranceau J, Oxland T. Spinal stability and intersegmental muscle forces. A biomechanical model. Spine (Phila Pa 1976) 1989;14:194-200. 
13. Magee DJ. Orthopedic physical assessment. 5th ed. Philadelphia: Elsevier Health Sciences; 2008.

14. Kwon JH, Cho MJ, Park MC, Kim SY. Cervical stabilization exercise using the Sling system. J Korean Acad Orthop Man Ther 2002;8:57-71.

15. Moon SB, Lee WJ, Hong CB, Kim KJ. Effects of cervical extension exercise and Mckinzie exercise on the pain and cervical muscle strength in patients with cervicalgia. Korea J Sports Sci 2007;16:687-98.

16. Chiu TT, Lam TH, Hedley AJ. A randomized controlled trial on the efficacy of exercise for patients with chronic neck pain. Spine (Phila Pa 1976) 2005;30:E1-7.

17. Falla DL, Campbell CD, Fagan AE, Thompson DC, Jull GA. Relationship between cranio-cervical flexion range of motion and pressure change during the cranio-cervical flexion test. Man Ther 2003;8:92-6.

18. Boyd-Clark LC, Briggs CA, Galea MP. Muscle spindle distribution, morphology, and density in longus colli and multifidus muscles of the cervical spine. Spine (Phila Pa 1976) 2002;27: 694-701.

19. DeStefano LA. Greenman's principles of manual medicine. 4th ed. Philadelphia: Lippincott Williams \& Wilkins; 2011.

20. Gong WT, Cheun HJ, Lee KM. The effect of cervical stabilized exercise and joint mobilization on maximum muscle strength and static muscle endurance of cervical region. J Korean Data Inf
Sci Soc 2010;21:33-42.

21. Mayoux-Benhamou MA, Revel M, Vallee C. Selective electromyography of dorsal neck muscles in humans. Exp Brain Res 1997;113:353-60.

22. Olson LE, Millar AL, Dunker J, Hicks J, Glanz D. Reliability of a clinical test for deep cervical flexor endurance. J Manip Physiol Ther 2006;29:134-8.

23. Taimela S, Takala EP, Asklöf T, Seppälä K, Parviainen S. Active treatment of chronic neck pain: a prospective randomized intervention. Spine (Phila Pa 1976) 2000;25:1021-7.

24. Kim DH, Jeong MK, Yoon WY. Effects of cervical and lumbar stabilization exercise program on lumbar reposition sense and static, dynamic balance ability, lower back pain in degenerated disc disease patients. J Sport Leis Stud 2013;53:813-21.

25. Kim SH, Kwon BA, Lee WH. Effects of cervical spinal stabilization training in private security on chronic neck pain and cervical function, neck pain, ROM. Korean Secur Sci Rev 2010;25:89108 .

26. Lee HY. The effects of the cervical stabilizing exercise on cervical proprioception, shoulder strength and hand grip strength. Master thesis. Daegu: Daegu University; 2012.

27. Jung JM, Gu JS, Shin WS. The effect of different sitting postures on range of motion, strength and proprioceptive sense of neck. J Korea Acad-Ind Coop Soc 2012;13:2212-8. 